\title{
Anomalous Coronary Artery Found in the Syncopal Workup of an Elderly Man
}

\author{
Ronnie Oommen, MD, Thad Wilkins, MD, Stephen Y. Chen, MD, \\ and Vishal Arora, MD
}

Syncope, defined as a transient loss of consciousness, is seen in $1 \%$ of all visits to emergency departments and urgent care clinics in the United States. Syncope is categorized as cardiogenic, neurologic, or psychogenic. Anomalies of the coronary arteries are rare, and anomalous coronary arteries present as syncope more often in the young than in the elderly; syncope rarely occurs in patients 65 years of age and older. There are 2 major variants of coronary anomalies. In the first variant, the left main coronary artery arises from the right aortic sinus. In the second variant, the right coronary artery arises from the left aortic sinus. The risk of sudden death is higher in patients with the left coronary artery arising from the right aortic sinus. We present a case of an anomalous coronary artery discovered during the syncopal workup in a 66-year-old man because no such cases have been published in the United States. We will discuss the management of anomalous coronary arteries as well as a systematic approach to the diagnosis and management of syncope. (J Am Board Fam Med 2012;25:541-546.)

Keywords: Anomalous Coronary Arteries, Case Reports, Elderly, Syncope

A 66-year-old white man presented to our family medicine clinic after experiencing syncope. The day before his presentation, he reported driving home from work when he experienced chest heaviness, followed by a sensation that he was going to "pass out." He slowed his car and pulled into a parking lot. Then he experienced a brief loss of consciousness. A few minutes after regaining consciousness, his chest heaviness subsided and completely resolved. He denied any seizure activity, tongue biting, or urinary or fecal incontinence. His medical history was significant for hypertension, Parkinson disease, and erectile dysfunction. His medications included carbidopa/levodopa, pramipexole, and vardenafil.

During examination, he was alert, awake, and oriented to person, place, and time. His vital signs

This article was externally peer reviewed.

Submitted 18 November 2011; revised 1 February 2012; accepted 7 February 2012.

From the Department of Family Medicine, Medical College of Georgia, Augusta.

Funding: none.

Conflict of interest: none declared.

Corresponding author: Thad Wilkins, MD, Department of Family Medicine, 1120 Fifteenth Street, HB-4032, Medical College of Georgia, Augusta, GA 30912 (E-mail: twilkins@georgiahealth.edu). included a heart rate of 85 beats per minute, supine blood pressure of 132/82 mm Hg, standing blood pressure of 118/78 $\mathrm{mm} \mathrm{Hg}$, and pulse oximetry of $99 \%$ on room air. No carotid bruits or murmurs were noted during auscultation. The patient had a resting tremor of his left hand and cog-wheel rigidity in the bilateral upper extremities. His gait was normal and his neurologic examination showed normal strength, sensation to light touch, and deep tendon reflexes.

His electrocardiogram showed normal sinus rhythm with nonspecific T-wave flattening. His hemoglobin level was $13.9 \mathrm{~g} / \mathrm{dL}$ (normal, 14-18.0 $\mathrm{g} / \mathrm{dL}$ ), his glucose level was $110 \mathrm{mg} / \mathrm{dL}$ (normal, $74-160 \mathrm{mg} / \mathrm{dL}$ ), and his sodium, potassium, and calcium levels were normal. His cardiac enzymes included Troponin I, $0.010 \mathrm{ng} / \mathrm{mL}$ (normal, 0.020$0.060 \mathrm{ng} / \mathrm{mL}$ ) and creatine phosphokinase, $76 \mathrm{u} / \mathrm{L}$ (normal, 51-294 u/L). His chest radiograph showed no acute cardiopulmonary process.

The patient was admitted to our family medicine inpatient service with telemetry monitoring. Myocardial infarction was ruled out with serial cardiac enzymes. A dipyridamole exercise myocardial perfusion scan was performed and was within normal limits. A transthoracic echocardiogram noted an 
Figure 1. Normal coronary anatomy and anomalous origin of left coronary artery (LCA). A: The normal origins of the coronary arteries are shown, with the LCA arising from the left sinus of Valsalva. B: The anomalous origin of the LCA from the right sinus of Valsalva is depicted showing 2 variants. The "benign" variant (dashed lines) runs in the septum anterior to the pulmonary artery (PA), whereas the "malignant" version runs posterior to the PA and between the annuli of the aorta and PA, creating a risk for compression of the LCA and compromise of blood flow to the myocardium. RCA, right coronary artery; LAD, left anterior descending artery. Artwork provided by Steven J. Harrison, PhD, CMI; Chairman of Medical Illustration Graduate Program, Georgia Health Sciences University.

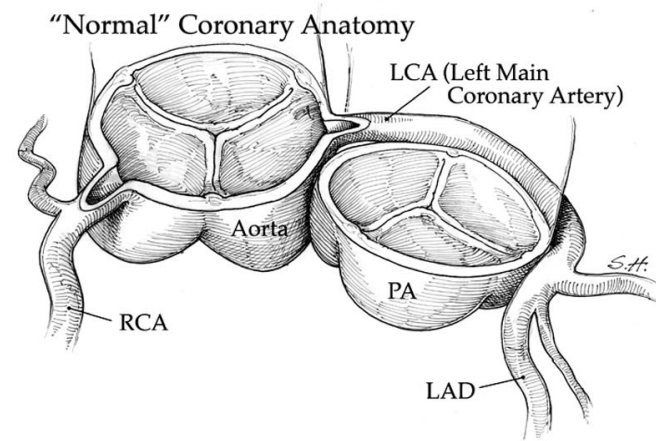

A.

ejection fraction of $50 \%$, inferior and apical hypokinesis, and impaired relaxation of the left ventricle with diastolic function. Cardiology was consulted and recommended that he undergo a cardiac catheterization.

His cardiac catheterization did not reveal any significant coronary artery disease but did show a left main coronary artery arising anomalously from the right coronary sinus. His coronary artery circulation was right dominant, with the right coronary artery supplying the posterior descending artery. In addition, the inferolateral wall was supplied by several large-caliber posterolateral branches arising from the right coronary artery. A coronary computed tomography angiogram was performed and showed an anomalous origin of the left main coronary arising off the right coronary sinus of Valsalva, running an oblique intra-arterial course anterior to the pulmonary artery and ascending aorta (Figures 1 to 3 ). He was referred to cardiothoracic surgery for further evaluation, and the surgeon did not recommend surgical repair because they felt the surgical risks outweighed the benefits of surgery. The patient was discharged from the hospital and continued taking his medications plus aspirin and propranolol. The anomalous coronary artery in this patient was the benign variant and was discovered during the workup of syncope. Since he had lived until the

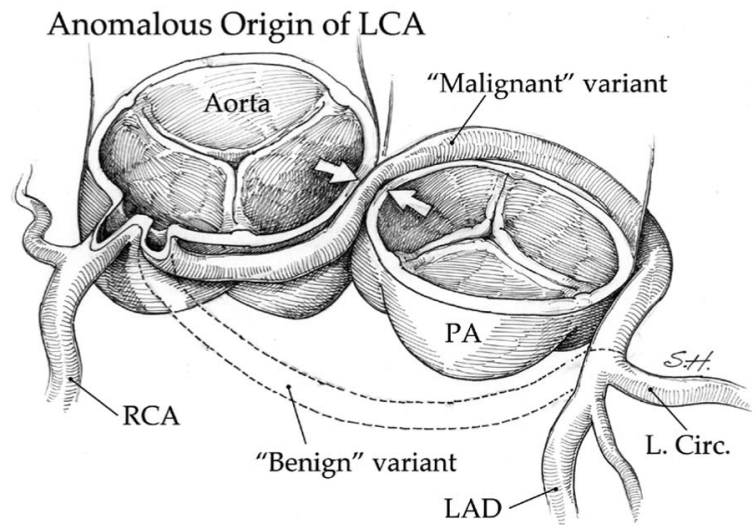

B.

age of 66 without prior cardiac symptoms, it is unlikely that his anomalous coronary artery was symptomatic or caused his syncope.

Figure 2. Cardiac computed tomography scan (3dimensional reconstruction). The pulmonary artery (PA) was selectively removed from volume rendering images to demonstrate an interarterial course of the left main (LM) coming off the right coronary cusp with the right coronary artery (RCA). LAD, left anterior descending artery; LCx, left circumflex artery.

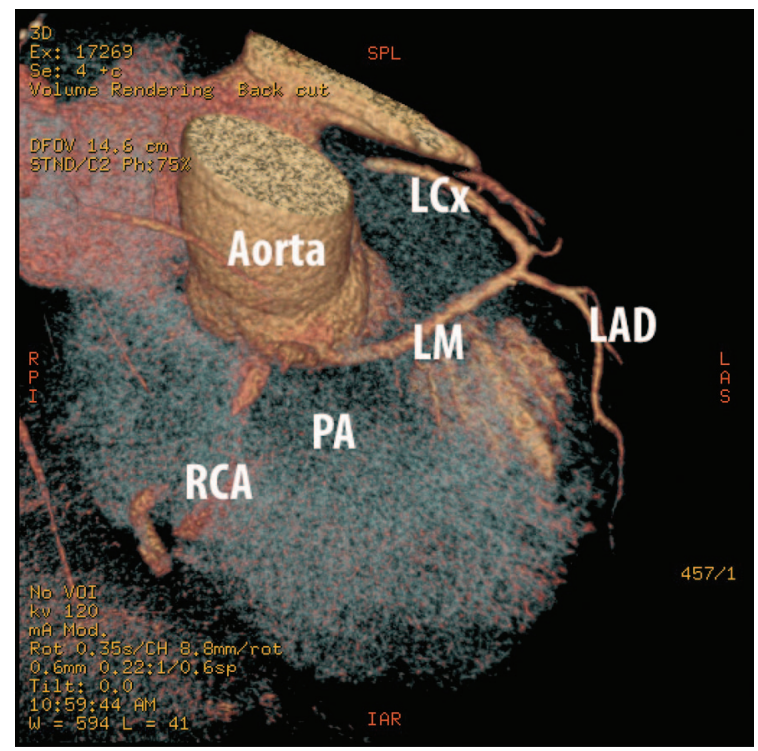


Figure 3. Cardiac computed tomography scan. Left main artery (LM) and right coronary artery (RCA) originated from the right coronary cusp. The course of the LM between the aorta (Ao) and the pulmonary artery (PA) is seen clearly. DscAo, descending aorta; $\mathrm{LA}$, left atrium; LV, left ventricle; PV, pulmonary vein.

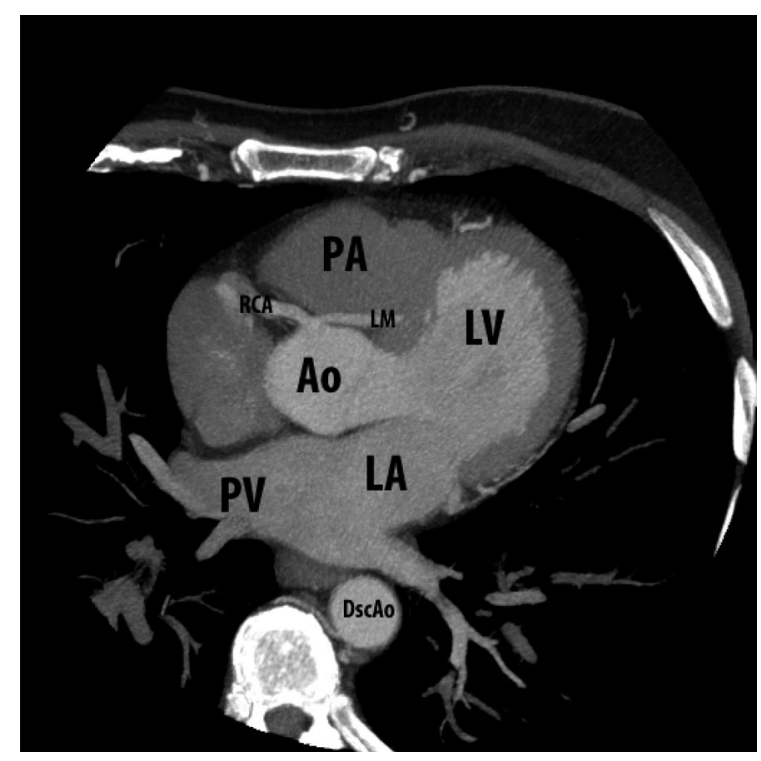

\section{Workup of Syncope}

Syncope, defined as a transient loss of consciousness, is seen in $1 \%$ of all emergency department visits and urgent care clinics in the United States. ${ }^{1}$ Syncope is categorized as cardiogenic, neurologic, or psychogenic. In patients older than 45 years of age, neurocardiogenic, structural heart disease, and psychogenic manifestations are common causes of syncope. ${ }^{2,3}$ Syncope presents with a wide variety of symptoms associated with a transient loss of consciousness. The diagnosis often is complicated by the fact that the patient is asymptomatic at the time of evaluation. Although the cause of syncopal episodes most often are secondary to benign conditions such as neurocardiogenic reflex, the goal of a thorough and systematic evaluation is to identify serious and life-threatening conditions. A systematic approach to a patient with syncope ensures a complete and thorough workup. Patients with a clinical or electrocardiographic history suggestive of arrhythmia, severe anemia, electrolyte abnormalities, family history of sudden death, older age, and history of severe structural heart or coronary artery disease are considered high risk and hospital admission is recommended. ${ }^{4,5}$ Patients younger than 50 years of age with no history of cardiovas- cular disease and a normal electrocardiogram and cardiovascular examination are considered low risk and outpatient evaluation is recommended.,

At the initial evaluation, a detailed medical history and physical examination, including a careful medication review, measurement of orthostatic vital signs, and bilateral brachial blood pressure measurements should be obtained. The history and physical examination are crucial; studies have shown a diagnostic utility of $75 \%$ to $85 \%$ in patients with syncope. ${ }^{6}$ Initial diagnostic testing typically includes an electrocardiogram in all patients and complete blood count, electrolytes, and glucose measurement as indicated. ${ }^{7}$ The electrocardiogram serves as a noninvasive tool in the detection of cardiac etiologies, for example, arrhythmia, myocardial ischemia, or ventricular hypertrophy. ${ }^{8}$ The initial findings from the history, physical, and laboratory evaluation will help to narrow the focus of evaluation into cardiogenic, neurologic, and psychogenic (Figure 4).

If cardiac ischemia is suspected based on the history and electrocardiogram, cardiac enzymes should be obtained and an evaluation for cardiac ischemia should be initiated (eg, cardiac stress testing or cardiac catheterization). In patients with history of cardiac disease or abnormal findings during the initial evaluation, and echocardiogram should be obtained and a Holter monitor used. Echocardiography may identify structural heart disease as well as identify left ventricular systolic dysfunction. ${ }^{9}$ A Holter monitor may identify arrhythmias such as supraventricular tachycardia or paroxysmal atrial fibrillation. Electrophysiologic studies should be considered in patients with probable arrhythmia-induced syncope. In the absence of structural or cardiac arrhythmias, tilt table testing might be useful to assess neurocardiogenic causes.

Neurogenic and psychogenic causes of syncope should be considered in the absence of any cardiogenic cause or based on initial evaluation. Noncontrasted computed tomography of the brain is often an initial evaluation of a noncardiogenic cause for syncope, followed by carotid duplex ultrasonography. Initial testing will help distinguish primary versus secondary autonomic failure in the neurological evaluation of syncope. In the absence of neurological findings, it is important to consider psychogenic causes such as factitious disorders. ${ }^{6} \mathrm{~A}$ neurology or psychiatry consultation may be helpful if the etiology is still unclear. Figure 3 shows an 
Figure 4. Algorithmic approach to the workup of syncope in adults.

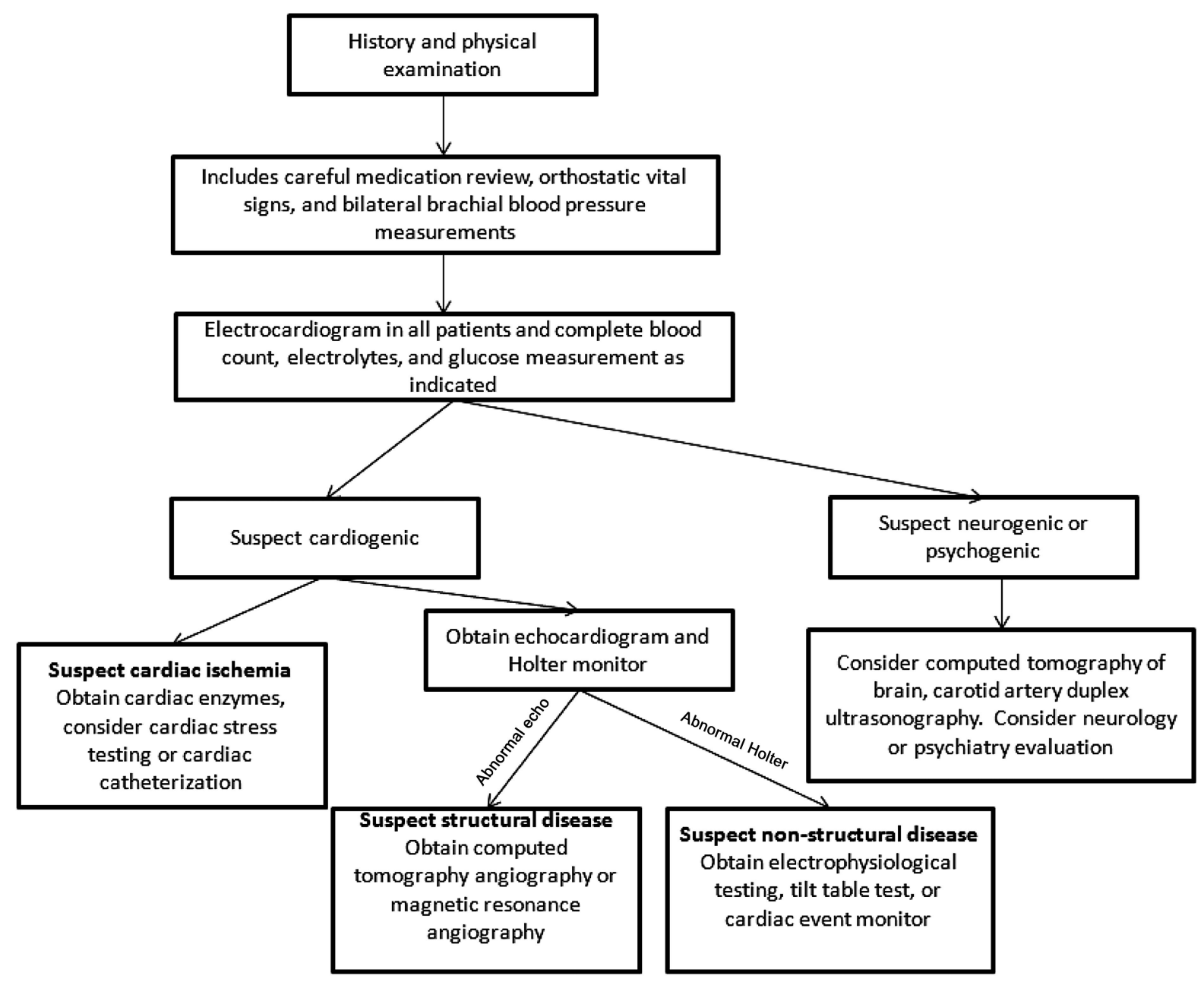

algorithmic approach to syncope in the adult patient. We suspected a cardiogenic cause of syncope in our patient on the basis of his abnormal electrocardiogram and echocardiogram, which prompted additional cardiac testing and led to the diagnosis of an anomalous origin of the coronary artery.

\section{Anomalous Origin of the Coronary Artery}

Anomalies of the coronary arteries are rare, with an incidence of $0.2 \%$ to $1 \%$, and anomalous coronary arteries present as syncope more often in the young than in the elderly; they rarely occur in patients $\geq 65$ years of age. ${ }^{2,3}$ There are 2 major variants of coronary anomalies. In the first variant, the left main coronary artery arises from the right aortic sinus, and in the second variant, the right coronary artery arises from the left aortic sinus. The risk of sudden death is higher for patients in whom the left coronary artery arises from the right aortic sinus. ${ }^{2}$
A recent case series of 151 adult patients with anomalous coronary arteries found that the mean reported age was 41 years, with $17 \%$ presenting with life-threatening conditions such as ventricular arrhythmia, syncope, or sudden death. ${ }^{10}$ Coronary artery with an anomalous origin represents a rare yet important cause of syncope. The left coronary artery or the left main artery typically arises from the left sinus of Valsalva and branches into the left anterior descending and left circumflex arteries. The right coronary artery usually arises from the right sinus of Valsalva. Rarely does the left coronary artery originate from the right sinus of Valsalva or the right coronary artery from the left sinus of Valsalva. The incidence of coronary artery anomalies was reported as much as $5.6 \%$ of the general population. ${ }^{11}$

The majority of coronary anomalies such as split right coronary artery or ectopic right coronary ar- 
tery from the right cusp are of no clinical significance. Coronary arteries with anomalous origins can result in episodic or obligatory myocardial ischemia and have been implicated in chest pain, syncope, myocardial ischemia, malignant ventricular arrhythmia, and sudden cardiac death. An anomalous left coronary artery from the pulmonary artery leads to obligatory ischemia with a $95 \%$ mortality rate during the first year of infancy. In comparison, anomalous aortic origin of a coronary artery may result in only episodic clinical symptoms and may not be discovered until adulthood.

The subsequent course of coronary artery can be interarterial (between the aorta and pulmonary artery), retroaortic, prepulmonic, or septal (beneath the right ventricular outflow tract), all of which predispose otherwise young, healthy individuals to dynamic myocardial ischemia or sudden cardiac death. The increased risk of sudden death may be because of external compression from great arteries, especially after exercise because exercise leads to the expansion of the aortic root and pulmonary trunk. In addition, the aberrant coronary artery may take an intramural course or have a slit-like ostium or acute angulation with a bend, further reducing the luminal diameter. According to the Sudden Cardiac Death Committee of the American Heart Association, coronary anomalies may have contributed to $19 \%$ of sudden cardiac deaths among athletes, ${ }^{12}$ second only to sudden death caused by hypertrophic cardiomyopathy. ${ }^{13}$

The diagnosis of anomalous origin of a coronary artery often has been established postmortem. In patients with suspected anomalous coronary arteries, echocardiography may establish the diagnosis, but the predictive value remains controversial. ${ }^{14,15}$ Coronary angiography is generally useful to establish the presence of coronary anomalies. However, the exact course of the aberrant coronary artery may not be visualized and determined easily. A coronary computed tomography angiogram provides an excellent depiction of coronary ostia and the anatomic course of the artery. ${ }^{16,17}$ Coronary magnetic resonance angiography also is being used increasingly because of excellent anatomic delineation and avoidance of radiation exposure, especially in young adults with suspected coronary anomalies. ${ }^{18}$

The presence of anomalous course of coronary artery between the aorta and pulmonary artery in young adults remains the greatest risk for an ad- verse event with or without clinical symptoms. ${ }^{19}$ Surgical intervention is indicated when the left coronary arteries arise from the right sinus and course between the aorta and pulmonary artery. Surgical repair is indicated, with documented myocardial ischemia when the arteries course between the aorta and pulmonary artery or intramurally. When the right coronary artery arises from the left sinus, surgical repair also is indicated when there is unexplained inducible ischemia in the right coronary artery territory or when it courses between the aorta and pulmonary arteries. ${ }^{20}$ Surgical revascularization with coronary artery bypass grafting, unroofing (marsupialization), or coronary reimplantation has favorable results, but coronary artery bypass grafting is deemed less desirable because of the presence of competitive flow. Coronary artery stenting also has shown good short-term results and could be the only option for critically ill patients. $^{21,22}$

\section{Conclusions}

Anomalous coronary artery, though a rare cause of syncope, can be missed easily without a proper systematic approach. This case report describes an anomalous coronary artery discovered during the syncopal workup of an elderly man. Syncope is a common cause of emergency department visits. The appropriate approach to evaluation includes a complete history and physical examination and electrocardiogram for all patients. Consider complete blood count, glucose measurement, and electrolytes as indicated. Additional testing may include echocardiogram, Holter monitor use, cardiac ischemia valuation, tilt table testing, and electrophysiologic study. Echocardiography is a noninvasive tool that helps to assess ventricular function and wall motion abnormalities.

\section{References}

1. Benditt DG, Can I. Initial evaluation of "syncope and collapse" the need for a risk stratification consensus. J Am Coll Cardiol 2010;55:722-4.

2. Kumpf M, Sieverding L, Gass M, Kaulitz R, Ziemer G, Hofbeck M. Anomalous origin of left coronary artery in young athletes with syncope. BMJ 2006; 332:1139-41.

3. Kastanis P, Marti V, Gotsanayidou M. Anomalous left coronary artery arising from the right sinus of Valsalva causing angina pectoris. J Cardiovasc Med 2009;10:574-5. 
4. Moya A, Sutton R, Ammirati F, et al. Guidelines for the diagnosis and management of syncope (version 2009). Eur Heart J 2009;30:2631-71.

5. Shen WK, Decker WW, Smars PA, et al. Syncope Evaluation in the Emergency Department Study (SEEDS): a multidisciplinary approach to syncope management. Circulation 2004;110:3636-45.

6. Kanjwal MY, Grubb BP. Evaluation of syncope. Curr Cardiol Rep 2005;7:329-35.

7. Strickberger SA, Benson DW, Biaggioni I, et al. AHA/ACCF scientific statement on the evaluation of syncope: from the American Heart Association Councils on Clinical Cardiology, Cardiovascular Nursing, Cardiovascular Disease in the Young, and Stroke, and the Quality of Care and Outcomes Research Interdisciplinary Working Group; and the American College of Cardiology Foundation In Collaboration With the Heart Rhythm Society. J Am Coll Cardiol 2006;47:473-84.

8. Ammirati F, Colivicchi F, Santini M. Diagnosing syncope in clinical practice. Implementation of a simplified diagnostic algorithm in a multicentre prospective trial-the OESIL 2 study (Osservatorio Epidemiologico della Sincope nel Lazio). Eur Heart J 2000;21:935-40.

9. Sarasin FP, Junod AF, Carballo D, Slama S, Unger PF, Louis-Simonet M. Role of echocardiography in the evaluation of syncope: a prospective study. Heart. 2002;88:363-7.

10. Yau JM, Singh R, Halpern EJ, Fischman D. Anomalous origin of the left coronary artery from the pulmonary artery in adults: a comprehensive review of 151 adult cases and a new diagnosis in a 53-yearold woman. Clin Cardiol 2011;34:204-10.

11. Angelini PMD, Velasco JAMD, Flamm SMD. Coronary anomalies: incidence, pathophysiology, and clinical relevance. Circulation 2002;105:2449-54.

12. Maron BJ, Thompson PD, Puffer JC, et al. cardiovascular preparticipation screening of competitive athletes. A statement for health professionals from the Sudden Death Committee (clinical cardiology) and Congenital Cardiac Defects Committee (cardiovascular disease in the young), American Heart Association. Circulation 1996;94:850-6.

13. Maron BJ, Carney KP, Lever HM, et al. Relationship of race to sudden cardiac death in competitive athletes with hypertrophic cardiomyopathy. J Am Coll Cardiol 2003;41:974-80.

14. Davis JA, Cecchin F, Jones TK, Portman MA. Major coronary artery anomalies in a pediatric population: incidence and clinical importance. J Am Coll Cardiol 2001;37:593-7.

15. Frommelt PC, Frommelt MA, Tweddell JS, Jaquiss RDB. Prospective echocardiographic diagnosis and surgical repair of anomalous origin of a coronary artery from the opposite sinus with an interarterial course. J Am Coll Cardiol 2003;42:148-54.

16. Schmid M, Achenbach S, Ludwig J, et al. Visualization of coronary artery anomalies by contrast-enhanced multi-detector row spiral computed tomography. Int J Cardiol 2006;111:430-5.

17. Singh Nijjar P, Parameswaran A, Amanullah AM. Evaluation of anomalous aortic origins of the coronaries by 64-slice cardiac computed tomography. Rev Cardiovasc Med 2007;8:175-81.

18. McConnell MV, Ganz P, Selwyn AP, Li W, Edelman RR, Manning WJ. Identification of anomalous coronary arteries and their anatomic course by magnetic resonance coronary angiography. Circulation 1995;92:3158-62.

19. Basso C, Maron BJ, Corrado D, Thiene G. Clinical profile of congenital coronary artery anomalies with origin from the wrong aortic sinus leading to sudden death in young competitive athletes. J Am Coll Cardiol 2000;3 5:1493-501.

20. Warnes CA, Williams RG, Bashore TM, et al. ACC/ AHA 2008 Guidelines for the Management of Adults with Congenital Heart Disease: a report of the American College of Cardiology/American Heart Association Task Force on Practice Guidelines (writing committee to develop guidelines on the management of adults with congenital heart disease). Circulation 2008;118:e714-833.

21. Doorey AJ, Pasquale MJ, Lally JF, Mintz GS, Marshall E, Ramos DA. Six-month success of intracoronary stenting for anomalous coronary arteries associated with myocardial ischemia. Am J Cardiol 2000;86:580-2.

22. Jaffe R, Shiran A, Gaspar T, Lewis BS, Halon DA. Primary stenting of an anomalous left main coronary artery with an interarterial course during cardiac arrest: imaging with CT angiography. Circ Cardiovasc Imaging 2009;2:351-2. 